\title{
Fibromialgia en Trabajo: explorando su Impacto en el Desempeño Laboral
}

\author{
Jesús Marrero-Centeno ${ }^{1}$, Ivonne Moreno-Velázquez² \& Israel Sánchez-Cardona ${ }^{3}$
}

Recibido: 19/05/2017 Aceptado: 16/01/2018

DOI:10.21772/ripo.v36n1a01

\begin{abstract}
Resumen
Esta investigación cualitativa exploratoria pretende examinar la experiencia de la fibromialgia en el ámbito laboral y cómo la misma incide en el desempeño de las personas. Utilizamos el Modelo de Demandas y Recursos Laborales para entender cómo los recursos (laborales y personales) y las demandas afectan la efectividad en el trabajo de las personas con fibromialgia. Siete pacientes con fibromialgia residentes en Puerto Rico participaron en entrevistas semiestructuradas. Los resultados apuntan que la fibromialgia tiene un efecto negativo en el desempeño de tareas, principalmente si estas requieren de esfuerzo físico o continuidad en una misma postura por largos periodos. Además, el ambiente laboral incide en la sintomatología y está a su vez en el desempeño de las funciones. Por último, se presentan una serie de recomendaciones enmarcadas en la flexibilidad, recursos personales y la educación, las cuales buscan minimizar los efectos que tiene la fibromialgia en el trabajo.
\end{abstract}

Palabras clave: fibromialgia, empleo, estrategias, modelo de demandas, recursos.

\section{Fibromyalgia at work: exploring its Impact on Job Performance}

\begin{abstract}
This qualitative exploratory research seeks to examine the experience of fibromyalgia in the work context and how it affects the performance of people diagnosed with this disease. The authors use the Demands and Labor Resources Model to understand how resources (work and personal) and demands affect the effectiveness of people with fibromyalgia at work. Seven patients with fibromyalgia living in Puerto Rico participated in semi-structured interviews. The results show that fibromyalgia has a negative effect on the performance of the participants' tasks, especially if they require physical effort or continuity in the same posture for long periods. Also, the work environment could affect the symptomatology of the disease and how it affects their performances. Finally, the authors present a series of recommendations framed in flexibility, personal resources and education, seeking to minimize the effects that fibromyalgia has on the job.
\end{abstract}

Keywords: fibromyalgia, employment, strategies, model of demands, resources.

En los últimos años el interés por investigar la 2011). Esta variedad de factores, particularmente la fibromialgia ha aumentado considerablemente, debido diversidad de síntomas que presenta la enfermedad, a múltiples factores entre los que destacan: (a) la falta trastoca las actividades cotidianas y laborales de las de conocimiento sobre el origen de la enfermedad; (b) personas que presentan el diagnóstico. Esto lleva a la diversidad de síntomas que presenta la enfermedad; que la fibromialgia altere la cotidianidad y el estilo de (c) la alta demanda de diagnósticos; (d) la ausencia vida de las personas (Briones, Ronda, \& Vives, 2014). de un tratamiento que la elimine; (e) el alto consumo de servicios de salud; y (f) el efecto en el desempeño laboral de las personas que padecen la enfermedad (Vicente, Terradillos, Capdevila, Ramírez \& López,

La fibromialgia es catalogada como un síndrome crónico que se caracteriza por dolor generalizado y difuso que contiene un sinnúmero

1 MA, Centro de Investigaciones Sociales, Universidad de Puerto Rico, Recinto de Río Piedras. E.mail: jesus.marrero2@upr.edu

2 PhD, Departamento de Psicología, Universidad de Puerto Rico, Recinto de Río Piedras. E-mail: ivonne.morenovelazquez@upr.edu

3 PhD, Programa de PhD Clínico, Universidad Carlos Albizu. E-mail: isanchez1@sju.albizu.edu

Nota: el presente artículo fue publicado en el 2017. Sin embargo, con el fin de mantener la periodicidad de la Revista, el número corresponde al primer semestre del año 2017

Cómo citar este arículo: Marrero-Centeno, J., Moreno-Velázquez , I. \& Sánchez-Cardona, I. (2017). El impacto de la Fibromialgia en el desempeño laboral: un estudio exploratorio sobre el rol de las demandas y los recursos. Revista Interamericana de Psicología Ocupacional, 36(2), 8-21. DOI:10.21772/ripo.v36n1a01 
de otros síntomas (National Institute of Arthritis and Musculoskeletal and Skin Diseases, 2014). Dentro de los síntomas se destacan: la sensibilidad al tacto o al presionar articulaciones o músculos, fatiga, dificultad para dormir, cansancio, dificultad para pensar con claridad, ansiedad o depresión, migraña, problemas digestivos, dolor pélvico, vejiga hiperactiva $\mathrm{y}$ trastornos temporomandibular (American College of Rheumatology, 2017).

La incidencia en los diagnósticos de fibromialgia, así como de dolores crónicos incapacitantes, ha aumentado. Vilá (2007) señala que en los Estados Unidos alrededor de 50 millones de personas padecen de algún tipo de dolor que les lleva a buscar la incapacidad, debido a las dificultades que presentan en poder mantener una vida habitual. En Puerto Rico, Millán (2005) informó que los dolores músculo-esqueléticos representan aproximadamente el $70 \%$ de las lesiones reportadas a la agencia que se encarga de promover el bienestar, en lo referente a los accidentes laborales. Esta es una cifra alarmante, ya que la mayoría de las personas que solicitan ayuda al sistema de compensación por accidentes del trabajo en Puerto Rico lo hacen como consecuencia de alguna lesión o dolencia muscular. Aunque no existen datos certeros de cuántos de estos casos corresponden a la fibromialgia, es posible que algún porcentaje de esa elevada cifra se relacione con casos en los cuales los dolores musculares podrían ser causados por la fibromialgia, teniendo en cuenta que las personas con este diagnóstico perciben su enfermedad con un alto nivel de discapacidad y una calidad de vida muy inferior en comparación con otras personas que presentan otros dolores crónicos (Burckhardt, Clark, \& Bennett, 1993).

Los constantes dolores en conjunto a la compleja y difusa sintomatología de la enfermedad, lleva a que las personas que presentan el diagnóstico se vean obligadas a rendir explicaciones por sus constantes ausencias o retrasos en el ámbito laboral (Briones et al., 2014). Además, la literatura evidencia que las personas diagnosticadas con fibromialgia presentan una mayor predisposición a incapacitarse o a estar desempleadas en comparación con personas con enfermedades músculo-esqueléticas (Rakovski, Zettel, \& Rutledge, 2012; Salido, Navarro, Judez \& Hortal, 2007). Según informan algunos estudios, la fibromialgia causa dificultades sustanciales en el desempeño laboral y en la calidad de vida de aquellas personas que presentan el diagnóstico (Liedberg, Burckhardt, \& Henriksson, 2006; Rojas, Zapata, Anaya \& Pineda, 2005). Por ejemplo, en Latinoamérica (ej., Colombia), al igual que en otros países a nivel mundial, se refleja cómo la sintomatología y los roles físicos minan la calidad de vida de las personas con fibromialgia (Rojas et al., 2005).

\section{Fibromialgia en el contexto laboral}

La literatura relacionada a cómo la fibromialgia incide en el ámbito laboral es limitada. Según la investigación disponible, las actividades laborales que requieran fortaleza o resistencia muscular (Henriksson, Liedberg, \& Gerdle, 2005; Mannerkorpi \& Gard, 2012) y tareas que requieran estar en una misma postura por largos periodos (Mannerkorpi \& Gard, 2012), serán las tareas más afectadas entre las personas que presentan fibromialgia. Algunos empleos que pueden incluir estas tareas son: personal de mantenimiento, construcción, jardinería, centros de llamadas, entre otros. Además, la investigación resalta que los síntomas que más reportan las personas con fibromialgia en el contexto laboral son: dolor, cansancio y fatiga (Henriksson et al., 2005; Briones et al., 2014).

Teniendo en cuenta lo anterior, este estudio tiene como objetivo examinar cómo la fibromialgia y su sintomatología inciden o afectan el desempeño de las personas diagnosticadas con fibromialgia que trabajan. Utilizamos el Modelo de Demandas y Recursos Laborales (Bakker \& Demerouti, 2013) como marco para examinar los efectos de la sintomatología (física y psicológica) de la enfermedad en el contexto laboral y cómo esto desencadena una serie de demandas laborales adicionales que afectan el desempeño del trabajo. A su vez, a través del modelo y las narrativas de las personas participantes pretendemos identificar recursos laborales y personales que ayuden a mitigar el efecto de las demandas en el contexto laboral.

\section{Modelo de Demandas y Recursos Laborales en el estudio de la fibromialgia}

Bakker \& Demerouti (2013) desarrollaron el Modelo de Demandas y Recursos para examinar las demandas y recursos laborales, y los efectos directos e indirectos que estos tienen sobre el estrés laboral y la motivación. Este modelo incluye dos grandes categorías: demandas y recursos laborales. Definen las demandas laborales como "aquellos aspectos físicos, psicológicos, organizacionales o sociales del trabajo que requieren un esfuerzo sostenido y conllevan costos fisiológicos y psíquicos" (Bakker 
\& Demerouti, 2013, p.108). Cuando las personas perciben que las demandas son altas, es necesario ejercer mayor esfuerzo para alcanzar los objetivos del trabajo y prevenir una disminución del desempeño. Esto puede conllevar un costo físico y psicológico, el cual puede provocar, a su vez, fatiga e irritabilidad (Schaufeli \& Taris, 2014).

Por otra parte, definen los recursos laborales como "los aspectos físicos, psicológicos, organizacionales o sociales del trabajo que pueden: (a) reducir las exigencias del trabajo; (b) ser decisivos en la consecución de los objetivos del trabajo; o (c) estimular el crecimiento personal, aprendizaje y el desarrollo" (Bakker \& Demerouti, 2013, p.108). El modelo contempla además el rol de los recursos personales, los cuales consisten en "autoevaluaciones positivas vinculadas a la resiliencia referidas a la percepción de la capacidad propia para controlar e influir en el entorno" (Bakker \& Demerouti, 2013, p.109).

Los recursos personales tienen efectos en el bienestar de los trabajadores y pueden moderar la relación entre las características del puesto y el bienestar (Schaufeli \& Taris, 2014). De igual modo, sirven de vías para explicar la relación entre los recursos laborales y los aspectos asociados a la salud y bienestar (Xanthopoulou, Bakker, Demerouti \& Schaufeli, 2007). Las intervenciones basadas en recursos personales (ej., esperanza, optimismo, resiliencia y autoeficacia) son claves para fomentar el engagement y propiciar un mayor desempeño (Luthans, Youssef \& Avolio, 2007; Van Wingerden, Derks \& Bakker, 2015). Además, que las prácticas que redunden en el aumento de recursos personales tienden a incrementar los recursos laborales, lo que puede resultar en el logro de metas establecidas en el trabajo (Van Wingerden, et al., 2015).

Para el presente trabajo, los investigadores conceptualizaron la fibromialgia dentro del Modelo de Demandas y Recursos Laborales como característica adicional que presenta la muestra que participó del estudio. En las personas empleadas, la fibromialgia podría representar un mayor número de demandas o exigencias laborales, considerando que ya presentan unas limitaciones físicas y psicológicas inherentes a la enfermedad. La literatura académica constata cómo la fibromialgia puede provocar un desequilibrio entre los recursos y demandas laborales, debido a la severidad de los síntomas (Mannerkorpi \& Gard, 2012). En este sentido, la cronicidad de los síntomas acompañada de la sobrecarga laboral podría llevar a estas personas a confrontar mayores dificultades en el desempeño de su trabajo. Para minimizar el efecto devastador de las demandas laborales en la salud y el desempeño de las personas con fibromialgia en el trabajo, es importante propiciar recursos laborales y personales. Mientras más recursos tengan disponible las personas, mayor probabilidad de reducir o mitigar las demandas y sus efectos (Bakker \& Demerouti, 2013), incluso en personas que presenten fibromialgia. Esto podría redundar en un mayor ajuste de la persona en su contexto laboral, favoreciendo un mayor desempeño y calidad de vida laboral.

Mannerkorpi \& Gard (2012) señalaron que las oportunidades dirigidas a variar o ajustar las tareas del trabajo o el ambiente del mismo, podrían influenciar a que las personas con fibromialgia continúen en el trabajo. De igual forma, las investigaciones enfatizan en la importancia de los recursos (particularmente el apoyo social) en pacientes con fibromialgia, para mitigar el impacto negativo de las demandas en el trabajo (Bossema et al., 2012). Por tanto, el planteamiento es que los recursos (laborales y personales) ayudan a afrontar y minimizar las demandas que puedan ser causadas por la enfermedad y el contexto laboral.

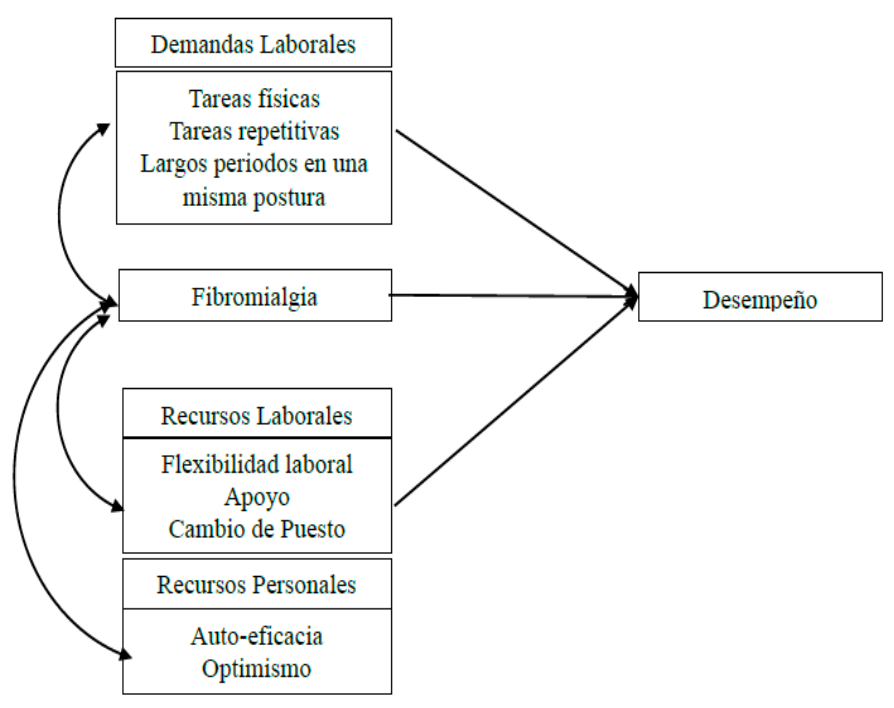

Figura 1. Modelo conceptual del estudio

\section{Método}

\section{Diseño}

Esta investigación es de tipo exploratorio, la cual es apropiada y utilizada con frecuencia cuando existe el interés de indagar sobre un constructo desde una nueva perspectiva teórica o cuando la información sobre el mismo es escasa. Esta investigación pretende examinar la fibromialgia desde una teoría proveniente de la investigación sobre el trabajo y las organizaciones (Modelo de Demandas-Recursos Laborales), lo cual resulta novedoso en la literatura 
sobre fibromialgia. Además, el enfoque de esta investigación es cualitativo, el cual busca explorar y entender cómo las personas o grupos significan un problema social o humano (Creswell, 2008). Los investigadores trabajaron con la técnica investigativa de entrevista semiestructurada para la recopilación de las experiencias de las personas que presentaban fibromialgia en el trabajo.

\section{Muestra}

La muestra de este estudio estuvo integrada por siete participantes residentes de Puerto Rico: seis mujeres y un hombre. La mayoría de las personas que participaron fueron mujeres, lo que es cónsono con investigaciones previas que destacan que el diagnóstico de la fibromialgia es predominante en esta población (Blasco et al., 2006; Valls, 2008). Para participar de la investigación, las personas debían cumplir con los siguientes criterios de inclusión: (a) tener el diagnóstico de fibromialgia por algún reumatólogo o doctor en medicina; (b) estar bajo empleo a tiempo parcial o completo; (c) tener 21 años o más, con capacidad propia para consentir; y (d) hablar el idioma español, debido a que la entrevista se realizó en ese idioma. Además de los criterios de inclusión, las personas debían estar de acuerdo con las condiciones establecidas en la hoja de consentimiento, principalmente consentir a que la entrevista se grabara en audio.

Al momento de la entrevista, seis participantes tenían empleo a tiempo completo $(86 \%)$ y solo una de las personas participantes trabajaba a tiempo parcial (14\%). En cuanto al nivel académico, el predominante fue bachillerato (86\%): seis de las personas participantes poseían este grado universitario. Solo una persona informó que no contaba con estudios universitarios. El rango de edad fue de 23 a 58 años, con una mediana de 43 años. El rango de tiempo que las personas llevaban con el diagnóstico de la enfermedad fluctuó entre 7 meses a 23 años. Las siete personas participantes residían en el área metropolitana de Puerto Rico, la cual comprende la capital de la Isla (San Juan) y ciudades limítrofes. Esta es el área más poblada y de mayor actividad económica en la Isla.

\section{Instrumento}

Como parte del trabajo se desarrolló una guía de preguntas (véase, Tabla 1), que buscaba examinar cómo incide la fibromialgia en el contexto laboral, en particular si el desempeño laboral se veía afectado debido a la enfermedad. La guía incluyó 23 preguntas relacionadas a los siguientes aspectos: sintomatología de la enfermedad, tareas afectadas, ambiente laboral, apoyo de compañeros y supervisores, modificaciones realizadas por los empleados con la condición, recursos personales y futuro laboral. El tiempo aproximado que le tomaba a las personas participantes completar la entrevista fluctuaba entre 45 a 55 minutos. La guía de la entrevista fue evaluada antes de su administración por dos jueces, académicos de la Universidad de Puerto Rico, y quienes se especializan en temas de salud ocupacional. Esta evaluación tuvo el propósito de determinar la precisión de las preguntas y si éstas podrían contribuir a recopilar los datos requeridos para este trabajo investigativo.

\section{Tabla 1}

\section{Ejemplo de la Guía de Preguntas}

\section{Guía de preguntas}

$1 \quad$ ¿Cuáles son los síntomas de la enfermedad que te afectan en el contexto laboral?

2 ¿De qué manera se ve afectado el desempeño de las tareas que realizas en el trabajo?

¿El ambiente psicológico (ej. relaciones de

3 compañeros, contexto en el que se da el trabajo) que permea en tu trabajo, afecta de alguna manera los síntomas de tu enfermedad?

$4 \quad$ ¿Ha recibido apoyo de algún compañero?

5 ¿Qué tipo de modificaciones o cambios se han realizado en tu trabajo?

¿Qué características personales le han ayudado a

6 sobrellevar la enfermedad? (ej. autoestima, optimismo, etc.)

7 ¿Cómo visualiza su futuro laboral?

\section{Procedimiento}

Este estudio contó con la aprobación del Comité Institucional para la Protección de Sujetos Humanos en la Investigación (CIPSHI) de la Universidad de Puerto Rico, Recinto de Río Piedras. Para reclutar la muestra de esta investigación, los autores utilizaron la técnica de bola de nieve. A través de anuncios en Internet y redes sociales (primordialmente Facebook), las personas interesadas en participar contactaron a alguno de los investigadores. Al establecerse el contacto con la posible persona participante, el investigador principal verificaba que esta cumpliera con los criterios de inclusión y le informaba sobre la hoja de consentimiento. El documento detallaba el propósito de la investigación, aspectos relacionados a la confidencialidad y el manejo de datos. También especificaba los posibles riesgos de la investigación que están asociados con cambios en el estado anímico. Con tal de minimizar los riesgos a las personas interesadas en participar el investigador principal, quien realizó las entrevistas, hacía hincapié 
en que la participación era voluntaria, y que podían finalizar su participación en cualquier instante. Si la persona participarte necesitara alguna intervención psicológica, el investigador principal podría referirle a una clínica psicológica de la Universidad de Puerto Rico. Esta clínica estaba al tanto de la investigación y su posible intervención. Por otra parte, las personas no recibieron ningún tipo de compensación. De estar de acuerdo con la hoja de consentimiento, se procedía a fijar la fecha, la hora y el lugar de la entrevista. La Universidad de Puerto Rico, Río Piedras proveyó un cuarto de estudio para realizar las entrevistas, aunque las personas participantes contaban con la prerrogativa de establecer donde querían ser entrevistados.

En esta investigación no se delimitó alguna zona específica de Puerto Rico para reclutar los participantes, dado que la prevalencia de la fibromialgia tiende a ser baja. Investigadores en países norteamericanos y europeos (Briones et al., 2014; Lawrence et al., 2008) han establecido entre un 2\% - 4\%. Por su parte, León y Loza (2015) indican que la prevalencia de la enfermedad a nivel internacional podría fluctuar entre $2 \%$ a $11.2 \%$.

\section{Análisis de datos}

El análisis de datos requirió grabar y transcribir todas las entrevistas verbatim para su posterior análisis. Para ello, los investigadores establecieron una guía de categorías y sus respectivas definiciones a priori a partir de los hallazgos de investigaciones previas y del modelo de investigación (véase Figura 1). La Tabla 2 presenta las categorías de análisis y sus definiciones. A pesar de contar con las categorías a priori, no se descartó la posibilidad de integrar nuevas categorías durante el proceso de análisis.

Los investigadores utilizaron el programa para análisis de datos cualitativos NVivo 11 para realizar las codificaciones. El investigador principal realizó el proceso de codificación inicial, el cual luego fue revisado por los coinvestigadores. Durante la codificación emergieron dos nuevas categorías de análisis: (1) Recomendaciones (sugerencias que ofrecieron las personas participantes respecto al manejo de la enfermedad) y (2) Enfermedades Conjuntas (enfermedades que presentan las personas en conjunto con la fibromialgia). Además, como parte del proceso de codificación y análisis de los datos, el investigador principal generó varias subcategorías emergentes (la Tabla 3, resume las características categorías y subcategorías utilizadas en esta investigación). Como parte del proceso de análisis de datos, los investigadores se encargaron de examinar las experiencias que compartieron las personas participantes a lo largo de las entrevistas. Del mismo modo, identificaron las tendencias en la que estas personas mencionaban o hacían alusión a ciertas categorías de análisis. Por último, evaluaron la consistencia y la diversidad en las respuestas de cada una de las personas participantes.

Tabla 2

Categorias y definiciones

\begin{tabular}{|c|c|}
\hline Categorías & Definiciones \\
\hline Clínico laboral & $\begin{array}{l}\text { Aspectos de la sintomatología } \\
\text { de la enfermedad en el entorno } \\
\text { laboral. }\end{array}$ \\
\hline Tarea laboral & $\begin{array}{l}\text { Actividades o tareas que realizan } \\
\text { las personas participantes en el } \\
\text { entorno laboral. }\end{array}$ \\
\hline Ambiente laboral & $\begin{array}{l}\text { Elementos que componen el } \\
\text { contexto en que se lleva a cabo la } \\
\text { tarea. }\end{array}$ \\
\hline Apoyo laboral & $\begin{array}{l}\text { Comprensión y entendimiento } \\
\text { que brindan los compañeros } \\
\text { y gerenciales respecto a la } \\
\text { enfermedad. }\end{array}$ \\
\hline $\begin{array}{l}\text { Modificaciones } \\
\text { laborales }\end{array}$ & $\begin{array}{l}\text { Cambios realizados por las } \\
\text { personas en su trabajo para poder } \\
\text { desempeñar las tareas. }\end{array}$ \\
\hline Futuro laboral & $\begin{array}{l}\text { Proyección futura que tiene la } \\
\text { persona participante con relación a } \\
\text { su trabajo. }\end{array}$ \\
\hline Recursos personales & $\begin{array}{l}\text { Características personales que } \\
\text { tienen las personas y que son } \\
\text { importantes para sobrellevar la } \\
\text { enfermedad. }\end{array}$ \\
\hline
\end{tabular}

Tabla 3

Resumen de categorías y subcategorías

\begin{tabular}{llll}
\hline Categorías & $\begin{array}{l}\text { Clínico- } \\
\text { laboral }\end{array}$ & Tarea-laboral & $\begin{array}{l}\text { Ambiente- } \\
\text { laboral }\end{array}$ \\
\hline Subcategorías & Físico & $\begin{array}{l}\text { Impacto adverso } \\
\text { Psicológico }\end{array}$ & $\begin{array}{l}\text { Perjudicial } \\
\text { Físico }\end{array}$ \\
& Apoas afectadas & $\begin{array}{l}\text { Perjudicial } \\
\text { Psicológico }\end{array}$ \\
\hline Categorías & $\begin{array}{l}\text { Apoyo- } \\
\text { laboral }\end{array}$ & $\begin{array}{l}\text { Modificaciones- } \\
\text { laborales }\end{array}$ & $\begin{array}{l}\text { Recursos- } \\
\text { personales }\end{array}$ \\
\hline Subcategorías & Companeros & Positivas & \\
& Gerencial & Futuras & \\
\hline Categorías & $\begin{array}{l}\text { Futuro- } \\
\text { laboral }\end{array}$ & Recomendaciones & $\begin{array}{l}\text { Enfermedades } \\
\text { Conjuntas }\end{array}$ \\
\hline \multirow{5}{*}{ Subcategorías } & Continuo & & \\
& $\begin{array}{l}\text { Fuera del } \\
\text { trabajo }\end{array}$ & & \\
\hline
\end{tabular}




\section{Resultados}

A lo largo de las entrevistas las personas participantes argumentaron sobre cómo la fibromialgia les afecta y limita en su contexto laboral. Los resultados apuntan a que la enfermedad genera una serie de demandas laborales adicionales, las cuales afectan el desempeño. Por otra parte, se desprende de sus experiencias una serie de modificaciones, recursos laborales y personales que les ayudan a mitigar los efectos de la fibromialgia en el contexto laboral. De igual forma, se destaca la importancia del apoyo de compañeros y supervisores. En las entrevistas están reflejadas expresiones relacionadas a los temas incluidos en las siguientes categorías: Clínico-laboral, Tarea-laboral, Futuro-laboral, Modificaciones-laborales, Recursos-personales, y Recomendaciones. Por otra parte, las siguientes categorías: Apoyo-laboral, Ambiente-laboral, y Enfermedades Conjuntas, no fueron tan recurrentes a lo largo de las entrevistas, en comparación con las categorías antes mencionadas.

\section{Tarea-laboral}

A través de las subcategorías impacto adverso y tarea afectada, las personas participantes expresaron los efectos negativos de la enfermedad en las tareas que realizan en el trabajo. Con relación a la subcategoría Impacto Adverso, manifiestan cómo la fibromialgia limita la capacidad para efectuar las tareas con la misma rapidez y agilidad, lo cual podría generar una sobrecarga de trabajo y, posiblemente, llevar a una eventual disminución del desempeño en las actividades laborales.

"Trabajo en una computadora, no puedo ir a la rapidez que quisiera” (P3)

"Un análisis que podía hacer en media hora, ahora me toma una hora" (P2)

"En cuestión de antes yo era más ágil en coger cajas y coger cosas" (P4)

Respecto a la subcategoría Tareas Afectadas por la enfermedad, argumentan que las tareas que estén asociadas con estar en una misma postura (de pie o sentado) por largos períodos, realizar tareas repetitivas y realizar tareas que requieran esfuerzo físico, serán los tipos de tarea que más les afecten en el trabajo. Esto evidencia cómo la severidad de la sintomatología se agudiza al tener que lidiar con estos tipos de tareas. Así que estas tareas podrían resultar más demandantes para quienes tienen la enfermedad e incluso los podrían llevar a la incapacidad.
"Estoy todo el día escribiendo en una computadora, y a veces tengo las manos trincas" (P1)

"Se me adormecen las piernas por estar en una misma postura” (P3)

"Lo más que me molesta es cuando tengo que hacer algo más arriba de mis hombros" (P4)

\section{Clínico-laboral}

En la subcategoría Física se percibe cómo hacen hincapié en los constantes dolores que sienten en diversas zonas de su cuerpo, narran cómo estos dolores están presentes dentro de su jornada laboral. Además de los fuertes dolores, hacen mención de otros síntomas físicos, que de igual manera les afectan y limitan. Estos otros síntomas mencionados son la fatiga y el cansancio. Algunas de las citas que reflejan los múltiples síntomas que experimentan son las siguientes:

"Un día te duele el brazo, un día te duele la mano, un día te duele la rodilla, un día es el cuello, un día es la cadera..." (P2)

"A mí el más que me afecta es el de la fatiga" (P2)

"Mucho dolor, el dolor es bien fuerte... a veces las caderas, las piernas, la parte al lado de la columna vertebral" ( $\mathrm{P} 4)$

"Te digo es como si me oprimieran el botón de off (apagado), y me da un cansancio" (en referencia a cuando termina la jornada laboral; P4)

Por otro lado, en cuanto a la sintomatología psicológica, mencionan un cambio en el estado de ánimo. Queda evidenciado en sus argumentos que lo que califican como depresión, es en parte una consecuencia de la incapacidad que experimentan al no poder realizar una serie de actividades con la misma agilidad que antes del diagnóstico. Otro síntoma que manifestaron la mayoría de las personas participantes fue problemas de memoria. Respecto a ese tipo de problema, argumentaron que en ocasiones tenían dificultad para recordar y concentrarse en la realización de tareas.

\footnotetext{
"Sabes, no voy a ponerme a llorar, porque como te digo estuve mucho tiempo deprimida y me di cuenta que no voy para ningún lado con eso" (P3)
} 
"Me dio tan duro que me afectó el entusiasmo, me afectó todo... uno entra en depresión” (P7)

"También se estaba afectando mi memoria" (P2)

$$
\text { "Me afectó en recordar las cosas" }
$$

\section{Modificaciones-laborales}

Através delas transcripcionesdelas entrevistas, se evidencian los cambios o modificaciones que realizan las personas participantes con el propósito de minimizar los efectos nocivos de la enfermedad en su trabajo. Estas modificaciones podrían ser parte de los recursos laborales que presenta el modelo conceptual del estudio (véase figura 1) y que las personas con fibromialgia utilizan para minimizar el impacto. Dentro de las modificaciones positivas señalan que prefieren tomar un receso corto para minimizar los efectos de la sintomatología de la enfermedad que les afecta en la realización de sus tareas.

"Cuando ya siento que no puedo, me paro y camino... necesito estirarme” (P3)

"Me he dado cuenta que, cuando me levanto y le tiro un chistecito mongo a cualquiera de mis compañeros, eso es como un brain-wash" (cambio de mentalidad; P2)

En cuanto a posibles modificaciones futuras (subcategoría), la mayoría coincide en que cambiarían algún equipo de oficina por uno que sea ergonómico. Por ejemplo, los más mencionados son sillas, teclados y relocalización de equipos tecnológicos. A través de estos recursos ergonómicos sienten que pudieran aminorar ciertas cargas provocadas por la enfermedad. Además, contarían con un ambiente laboral (físico) apto a sus necesidades, lo que les permitiría realizar mejor sus tareas.

$$
\begin{aligned}
& \text { "Una silla, para estar cómoda" (P5) } \\
& \text { "Un teclado con pad" (en referencia a un } \\
& \text { teclado ergonómico; } \mathrm{P} 4)
\end{aligned}
$$

\section{Recursos personales y Recomendaciones}

Durante las entrevistas, las personas mencionaron una gran cantidad de recursos personales y ofrecieron una serie de recomendaciones que a su entender les ha ayudado a sobrellevar su enfermedad tanto a nivel laboral, como personal. En relación con los recursos personales, destacan la importancia de enfrentar los retos del diario vivir de forma positiva. Según informan en las entrevistas, al comienzo de la enfermedad les era difícil mantener un estado de ánimo optimista, pero con el tiempo se han dado cuenta que es necesario. Reconocen que estar inmersos en estados de ánimo enmarcados en la tristeza y melancolía no les ayuda a lidiar con sus problemas. Por otra parte, destacan que les ha ido mejor cuando asumen una actitud positiva ante las situaciones desventajosas de la enfermedad y que a través del tiempo han demostrado que pueden lograr sus metas laborales y personales.

"He adoptado la actitud de que tengo que estar positiva" (P2)

"Yo soy una persona súper optimista, y siempre trato de buscarle el lado positivo a las cosas" (P5)

"Pensar siempre positivo... pues tengo que vivir feliz” (P6).

Entre las recomendaciones que ofrecieron se encuentran: (a) fomentar la flexibilidad en el lugar de trabajo, (b) promover la educación sobre la enfermedad, (c) realizar ejercicio regularmente, (d) seguir una dieta balanceada, (e) visitar regularmente a profesionales de la salud, y (f) seguir las terapias o tratamientos asignados. Según sus experiencias estas recomendaciones, podrían ayudar a propiciar un mejor ambiente de trabajo y a contrarrestar la severidad de los síntomas.

"Yo diría que laboralmente en lo que se puede trabajar es en la cantidad de horas que se trabaja y la flexibilidad" (P2)

"Yo pienso que hace mucha falta lo que es la educación, tanto para el paciente, como para el empleado" (P5).

"Mientras haga ejercicios creo más resistencia. Lo dejo de hacer y me pongo fatal" (P6)

“Tú sabes psicólogos, psiquiatras que ayuden" (P1).

\section{Apoyo Laboral}

En cuanto al Apoyo-laboral, no fue tan recurrente como las categorías anteriores. Esto debido a que hubo tres participantes que prefirieron no informarle a su patrono o compañeros de trabajo su padecimiento de fibromialgia. Las razones que ofrecieron giran en torno a la posibilidad de que les limiten oportunidades de crecimiento dentro de la 
propia empresa.

En cuanto a quienes decidieron comunicar la enfermedad a sus supervisores, compartieron que han recibido de éstos comprensión y flexibilidad. Según expresaron, sus supervisores les han dado la confianza y el espacio para que expresen sus preocupaciones y molestias entorno a la enfermedad. Por otra parte, quienes reciben apoyo de sus compañeros señalaron sentirse comprendidos. Mencionaron que los compañeros les motivan a continuar trabajando y los asisten cuando necesitan realizar una tarea demandante (ej., mover cajas).

"Yo la he sabido llamar para decirle: estoy mareada, voy a llegar a trabajar, pero necesito un tiempo en lo que me siento mejor. Tranquila tomate tu tiempo (respuesta del supervisor)" (P2)

"Ellos entienden (superiores), saben que si tengo que ir a una cita, terapia o algo, pues han sido condescendientes" (P4)

"Cuando he llegado un poco deprimida, me dan aliento. Me dicen: Mira que si echa para adelante y eso pues ayuda a uno, porque te alegra el día. Ay pérate déjame como que meterle un poquito más al trabajo" (en referencia al aliento que recibe por sus compañeros; P3)

"Tengo que pedir ayuda: Mira bájame esta caja... Bendito ellos (compañeros) me ayudan, me dicen: Siéntate no hagas fuerza, no hagas esto, no hagas lo otro" (P4)

\section{Ambiente-laboral}

Con relación al ambiente físico estuvieron de acuerdo en que su ambiente físico era adecuado, solo tres participantes manifestaron que en ocasiones suele ser muy frío. Cuando el ambiente físico se torna demasiado frío, expresan experimentar mayor dolor.

"Horrible, Horrible (en referencia al frío), porque me afecta el movimiento" (P1)

"Si hace mucho frío, tengo que hablar con un compañero para que suba el aire, porque a veces me da un dolor..." (P4).

Por otra parte, seis participantes argumentaron que en ocasiones tienen que lidiar con demasiadas demandas laborales (ej., fechas de entregas de trabajo, múltiples tareas al mismo tiempo, sobrecarga de trabajo, etc.), lo que les causa tensión y estrés. Según lo expresado la exposición a ambientes altamente estresantes tiene el potencial de incrementar los síntomas. Esto representa que la sobrecarga de demandas laborales les llevará a experimentar mayor dolor y una sintomatología más aguda.

"Si hay mucho estrés y tensión en el trabajo la próxima semana yo voy a estar de cama” (P2)

"Pero ya cuando hay mucho estrés, ahí sí que no valgo un centavo" (P4)

"Cuando siento que tengo mucho estrés, empiezo a sentir como que la carga en la parte atrás del cuello, y la espalda” (P5)

\section{Futuro-laboral}

Todos los participantes presentaron una visión favorable del trabajo. La mayoría desea continuar trabajando y no contempla incapacitarse o retirarse en un futuro cercano. Algunas de las razones que expusieron para continuar laborando son que el trabajo les mantiene ocupados y activos. Además, mencionaron que necesitan los ingresos para costear ciertas necesidades familiares (ej., educación de los hijos, necesidades básicas). A su vez, la mayoría de las personas participantes no se limitan a su trabajo y se muestran abiertos a participar de otras experiencias laborales. "Incapacitarme no lo veo dentro del
panorama"( $\mathrm{P} 2)$

"Pero yo no lo veo (incapacitarse), yo no me voy a dar por vencida y punto" (P4)

Por otra parte, solo dos participantes manifestaron que consideran irse de su trabajo en un futuro cercano. Es importante reconocer que estas dos personas puntualizaron que se iban de su trabajo dada la proximidad de su jubilación y no a causa de la fibromialgia. Incluso llevan una gran cantidad de años trabajando, a pesar de contar con el diagnóstico de fibromialgia. Esto demuestra que han podido lidiar con su enfermedad en el contexto laboral.

"Dentro de un año retírame, porque me queda bien poquito para retirarme" (en relación a su futuro laboral; P1)

"Bueno, ya no me queda mucho. Yo tengo 58 (en referencia a la edad). Llevo 37 años trabajando" (P3)

\section{Enfermedades conjuntas}

Finalmente, una de las categorías que emergió 
del análisis fue Enfermedades Conjuntas. Relacionado a esta, las personas mencionaron otras enfermedades que presentan junto a la fibromialgia. Seis participantes expresaron padecer otras enfermedades adicionales entre las cuales destacan: el síndrome del túnel carpiano, artritis y neuropatía. Estas otras condiciones y su severidad podrían complicar más el panorama laboral para las personas con fibromialgia.

"Yo tengo 'carpal tunnel"” (síndrome del
túnel carpiano; P3 y P5)

"Después entonces me viene esto de la mala cesárea, después me empieza la fibromialgia, la artritis, la neuropatía y todo ha sido una tras de otra" (P6)

"La condición (fibromialgia) a mi me la diagnosticaron en conjunto con neuropatía" (P7)

Los resultados señalan que el día a día de las personas con fibromialgia puede ser difícil dentro de su entorno laboral. A su vez puede estar cargado de múltiples demandas o exigencias laborales, las cuales podrían ir en incremento si no se cuenta con los recursos necesarios. De igual forma, no resulta tarea fácil presentarse al escenario laboral padeciendo una gama de síntomas físicos y psicológicos, los cuales podrían ser limitantes en ciertas facetas del trabajo. Ahora bien, de acuerdo con lo expresado por estas personas, es posible continuar en el trabajo, máxime si éstos muestran una actitud positiva ante la enfermedad y en el trabajo se les provee los recursos necesarios. Estos recursos serán de gran ayuda ante las demandas laborales que enfrentan, las cuales se intensifican por el padecimiento de la condición.

\section{Discusión}

Los resultados de las entrevistas a las personas diagnosticadas con fibromialgia sugieren que esta enfermedad y su sintomatología generan unas demandas laborales adicionales, las cuales tienen unas consecuencias negativas sobre el desempeño laboral. Esta aproximación exploratoria inicial a través de las entrevistas, refleja que las tareas más afectadas son aquellas que se relacionan con ejecutar esfuerzo físico: trabajar con los brazos elevados, mover cajas o algún equipo pesado, entre otras tareas que requieran gran demanda física. De igual modo, las tareas que requieren una misma postura por espacio prolongado de tiempo tienden a verse afectadas. Estos resultados son cónsonos con los encontrados en la literatura revisada que destacan que las personas con fibromialgia muestran dificultad para realizar tareas o actividades del trabajo que involucre manejar objetos pesados y permanecer en la misma postura por largos periodos (Henriksson et al., 2005). Todo el esfuerzo que estas realizan en el trabajo será más marcado que el esfuerzo que realizan las personas sanas (Palstam et al., 2014).

En cuanto a los efectos físicos que presenta la fibromialgia, estas personas señalan que los síntomas físicos que más les afectan y les limitan en el escenario laboral son los dolores en diversas áreas del cuerpo (manos, pies, espalda, hombros, etc.), el cansancio y la fatiga. Relacionado a estos síntomas, el dolor está presente continuamente en el trabajo y se manifiesta según la intensidad de las tareas que realizan los empleados. De acuerdo con sus narrativas, el dolor mostró ser el síntoma principal. En cuanto al cansancio, las personas mencionaron que lo presentaban cuando la jornada laboral había culminado o estaba por culminar. Por último, la fatiga, se manifestaba cuando llevaban gran parte del tiempo en la ejecución de una tarea. En ocasiones, entrelazaban los conceptos de cansancio y fatiga. Lo que sí parece ser un hecho es que estos dos síntomas acompañan la jornada laboral de las personas con fibromialgia. Estos hallazgos son similares con estudios investigativos previos, en los cuales se ha observado que las limitaciones que impiden a los sujetos con fibromialgia desempeñarse normalmente en su trabajo se derivan del dolor y la diversidad en la sintomatología de la enfermedad (Briones et al., 2014; Juuso, Skär, Sundin, \& Söderberg, 2016; Rakovski et al., 2012).

Por otra parte, las personas que padecen la enfermedad destacaron el cambio en el estado de ánimo como el principal síntoma psicológico que presentan. Estas describieron las alteraciones en su estado anímico como depresión, caracterizada por sentimientos de angustia y tristeza. Según los resultados que se desprenden de las entrevistas, la enfermedad y sus limitaciones, se observa una alteración del estado anímico, lo que a su vez podría afectar el desempeño de las personas en su contexto laboral. La literatura reseña que las personas con fibromialgia presentan una mayor predisposición a padecer ciertos trastornos psicológicos como lo son la ansiedad y la depresión (Blasco et al., 2006; Kassam \& Patten, 2006). Cuando la depresión surge como uno de los síntomas de la fibromialgia podría posibilitar la reducción de la participación y el desempeño laboral 
(Kassam \& Patten, 2006; Rakovski et al., 2012). Es importante puntualizar y reconocer que la severidad de los síntomas limita la habilidad para realizar actividades laborales, por ende es muy difícil mantener un desempeño normal (Guymer, Littlejohn, Brand \& Kwiatek, 2016; Palstam \& Mannerkorpi, 2017; Sallinen, Kukkurainen, Peltokallio, \& Mikkelsson, 2009).

Un hallazgo relevante es que además de los efectos físicos y psicológicos que presentan las personas con fibromialgia, gran parte de la muestra presentaba otras enfermedades en conjunto con la fibromialgia. Dentro de las enfermedades que expresaron con mayor frecuencia se encontraban la artritis, el síndrome del túnel carpiano y la neuropatía. Muchas de las enfermedades crónicas están asociadas con niveles bajos de calidad de vida (McDonald, daCosta, \& Ullman, 2011).

Por otra parte, los resultados reflejan la importancia del ambiente psicológico en las personas con fibromialgia en su entorno de trabajo. Estas perciben que en ocasiones tienen que lidiar con demasiadas demandas laborales (p.ej., fechas de entregas de trabajo, realizar múltiples tareas al mismo tiempo, entre otras.). Dichas demandas a su vez generan tensión y estrés, lo cual desemboca en mayor cronicidad de la enfermedad. El estrés percibido es un predictor de los síntomas y del impacto de la fibromialgia (González, García, \& Landero, 2011). Estar expuesto a periodos estresantes incrementa la probabilidad de provocar un deterioro del bienestar de las personas, incluso aumenta la posibilidad de discapacidad laboral (Salido et al., 2007).

El problema de que las personas empleadas con fibromialgia se incapaciten es que podrían estar expuestas a otras problemáticas que trastoquen aún más su calidad de vida. Según Kool y Geenen (2012), el tener una baja educación, estar sin empleo y tener fibromialgia, son variables que se relacionan significativamente con el aislamiento social percibido. De hecho, en investigaciones académicas han encontrado que las personas con fibromialgia que trabajan tienen mejor salud que las que no trabajan y tienen la enfermedad (Palstam \& Mannerkorpi, 2017).

Según la información que se desprende de los resultados del estudio, la fibromialgia y la cronicidad de su sintomatología genera una serie de demandas adicionales, las cuales sumadas a las demandas propias del trabajo hará del mismo uno más difícil. Esto podría desembocar en un pobre desempeño, ya que las personas no cuentan con la misma agilidad para realizar las tareas (Briones et al., 2014). De hecho, según puntualizan otras investigaciones, el bienestar y la calidad de vida de las personas con fibromialgia va en detrimento. Dado a esta situación, es importante que los patronos reconozcan los efectos de la enfermedad y la importancia de poder propiciar ciertos recursos los cuales podrían reducir el efecto de las demandas en el contexto laboral. A continuación, los investigadores exponen una serie de recomendaciones que podrían ser los recursos necesarios para ayudar a las personas empleadas con fibromialgia a minimizar los efectos que tiene la enfermedad en el contexto laboral.

\section{Implicaciones prácticas}

\section{Recursos}

\section{Modificaciones en el ámbito laboral.}

Las personas mencionaron que para lidiar con la enfermedad en el contexto del trabajo les ha sido de beneficio tener la flexibilidad de tomar pequeñas pausas cuando llevan largos periodos realizando la misma tarea. Estas breves pausas les permiten estirar sus músculos y evitar algún entumecimiento de los mismos, lo que previene que experimenten un mayor dolor. Además, les posibilita despejar su mente al menos por unos minutos, para luego poder continuar con sus respectivas tareas. Algunas de estas personas mencionaron que durante ese tiempo hacen algún chiste, ríen, escuchan un poco de música relajante o simplemente se estiran. La flexibilidad en el trabajo que le permite a las personas poder emplear estas diversas maneras de lidiar con la enfermedad, ayuda a disminuir la posibilidad de confrontar sobrecarga laboral (Palstam, Gard \& Mannerkorpi, 2013). De igual forma ayuda a minimizar el dolor, les permite centrarse en sus respectivas tareas y continuar con su trabajo. Es una estrategia que fomentaría mantener un trabajo adecuado y sustentable para aquellas personas con fibromialgia (Bossema et al., 2012).

Otras modificaciones en el ámbito laboral podrían estar dirigidas a tener equipos ergonómicos, tales como: sillas, teclados, entre otros. El uso de estos equipos especiales permite reducir el impacto de la enfermedad en el trabajo y a su vez facilita que no se experimente tanto dolor. Las personas que participaron de este estudio vislumbran que en un futuro cercano podrían incorporar algunos equipos ergonómicos para mejorar su contexto laboral. Incluso, varias de estas recibieron el visto bueno de su patrono para proceder con la compra de una silla que le permitiera realizar el trabajo con mayor comodidad. 
Esta incorporación del equipo ergonómico al trabajo es importante, debido a que puede ser un recurso que permita a la persona aminorar las demandas de la fibromialgia en el trabajo.

Por otra parte, los patronos podrían implementar modificaciones en las condiciones de trabajo de las personas con fibromialgia. Algunas otras modificaciones que los patronos podrían implementar serían: (a) reducción de la jornada laboral; (b) evaluar la posibilidad de realizar trabajo desde el hogar; y (c) cambio de puesto. Estas recomendaciones van a la par con los hallazgos de Rakovski et al. (2012), quienes señalan que existe una probabilidad de que las personas con fibromialgia modifiquen las condiciones laborales y en ocasiones sus carreras para poder ajustarlas a su enfermedad. Para esto es importante el reconocimiento temprano del impacto de la fibromialgia en el trabajo, debido a que posibilita el manejo inmediato de la situación, lo cual a su vez ayuda a reducir el efecto perjudicial que podría provocar (Guymer et al., 2016). Es fundamental que quienes viven con fibromialgia soliciten modificaciones o cambios en el trabajo y que el patrono este informado sobre las responsabilidades $\mathrm{y}$ posibilidades de adquirir fondos que hagan del trabajo uno adecuado (Bossema et al., 2012).

Recursos Personales. Además de las diversas modificaciones en el trabajo, otro componente importante son los recursos personales. Este componente ha recibido poca atención de la literatura científica vinculada a la discapacidad laboral (Bossema et al., 2012). Aunque, los recursos personales podrían ser parte de las estrategias de afrontamiento que ayuden a mitigar los síntomas de pacientes y a su vez permitir que se mantengan productivos. A través de los recursos personales las personas con fibromialgia combaten los efectos de la enfermedad (p. ej., propiciando actividades que les brinden felicidad) (Kelley \& Clifford, 1997). Löfgren, Ekholm y Öhman (2006) enfatizan que mientras más se conozca sobre las estrategias de afrontamiento que ayudan a estas personas, mejores podrían ser los programas de rehabilitación.

Los recursos personales que reflejaron las personas iban dirigidos a ser optimistas y positivos ante las adversidades que enfrentan. Además, la mayoría ha asumido una actitud luchadora y autosuficiente en donde buscan vencer la enfermedad y a su vez demostrar que pueden cumplir los objetivos y metas trazadas. Esta actitud los mantiene activos en el escenario laboral, cumpliendo con sus tareas y metas profesionales. Incluso, algunos siguen luchando para propiciar nuevas experiencias laborales que les permitan seguir creciendo dentro de su entorno profesional. Esto es afín con los hallazgos de otros estudios en los cuales las mujeres con fibromialgia emplearon una serie de estrategias, por ejemplo, los pensamientos positivos y la búsqueda del disfrute de la vida (ej., disfrutar los placeres de la vida - comida, música, bebida, etc.) para afrontar la enfermedad (Löfgren et. al., 2006; Oldfield, MacEachen, MacNeill \& Kirsh 2017). Estas estrategias les ayudaron a permanecer trabajando, a pesar de contar con una gran cantidad de síntomas.

Los resultados de esta investigación relacionados a los recursos personales, así como lo observado en otras investigaciones, parece relevante al desarrollo de intervenciones dirigidas a fomentar optimismo, felicidad, resiliencia, autoeficacia, entre otras variables positivas destinadas a aumentar los recursos psicológicos positivos de las personas con fibromialgia. En investigaciones recientes se ha examinado cómo el capital psicológico (el cual se conforma por la eficacia, el optimismo, la esperanza y la resiliencia), se relaciona con el bienestar (YoussefMorgan \& Luthans, 2015; Luthans, Youssef-Morgan, Sweetman \& Harms, 2012). El desarrollo del capital psicológico y otros recursos personales podrían propiciar un mejor desempeño en el trabajo, mayor bienestar y mejores relaciones (Avey, Reichard, Luthans \& Mhatre, 2011; Luthans et al., 2012).

Educación. Respecto a la educación, consideramos que es primordial que la persona se eduque sobre la fibromialgia para que la conozca más a fondo y pueda educar a otras personas (amistades, compañeros, patronos). Además, el conocimiento sobre la enfermedad le permitirá mayor control sobre la misma y desarrollar ajustes necesarios para mejorar su calidad de vida. La Asociación Americana de Reumatología (2013) recomienda que los pacientes se eduquen y que estos a su vez puedan educar a otros. En el contexto de trabajo, es también de vital importancia que los patronos estén abiertos y dispuestos a conocer sobre la fibromialgia y cómo podrían atender a quienes presentan la misma.

El conocimiento de la enfermedad por los patronos favorece que estos ofrezcan los recursos necesarios para que las personas con fibromialgia ajusten su entorno laboral de una manera más saludable y productiva. Dentro de los recursos que pueden proveer a las personas empleadas con fibromialgia están: (a) flexibilidad laboral; (b) incorporar aspectos ergonómicos al ámbito laboral; (c) integrar a las personas con fibromialgia a equipos de trabajo para el desarrollo de ciertas tareas; (d) proveer membresías a 
gimnasios o algún lugar para que puedan ejercitarse; (e) ofrecer la oportunidad de realizar otra gama de tareas; y, (f) propiciar ambientes psicológicamente saludables. Estos ambientes saludables se enfocan en el balance vida-trabajo, la salud y seguridad, la capacitación, la participación, el crecimiento y desarrollo y el reconocimiento de las personas, con la comunicación como eje central ${ }^{4}$.

Todos los recursos antes mencionados podrían contribuir a que las personas empleadas con la enfermedad se sientan comprendidos por sus patronos. Observamos que varias investigaciones subrayan la importancia de que las personas con fibromialgia reciban comprensión, ayuda y apoyo en su ambiente laboral, para que puedan continuar laborando (Bossema et al., 2012; Juuso et al., 2016; Löfgren et al., 2006). Otro aspecto que influye a que estas personas permanezcan en su trabajo, es que suelen percibir su trabajo como significativo (Juuso et al., 2016; Palstam et al., 2013).

Es posible que algunos patronos no tengan los recursos necesarios para llevar a cabo algunas de las estrategias expuestas anteriormente. No obstante, el patrono puede evaluar cada una de las alternativas y conforme a las necesidades de las personas que emplea y los recursos que tiene disponibles, puede identificar la más efectiva para ambos. Es importante que las organizaciones fomenten el diálogo para atender estas situaciones de salud o cualquier asunto que busque mejorar la calidad de vida de estas personas.

\section{Limitaciones del estudio y futuras líneas de investigación}

Los resultados de este estudio deben interpretarse teniendo en cuenta algunas limitaciones. En primer lugar, la muestra del estudio fue pequeña y solo incluye la perspectiva de un grupo de participantes sobre su experiencia en el ambiente laboral y su padecimiento de fibromialgia. Esto limita la generalización de los resultados y existe la posibilidad de que no hayamos auscultado otros aspectos importantes sobre el vínculo de la fibromialgia y el desempeño laboral. Un segundo aspecto que resaltar es que, a pesar de las garantías de confidencialidad, existe la posibilidad de que las personas participantes se limitaran en compartir información y ocultaran aquella que consideraran que los podría poner en desventaja con sus empleadores. En tercer lugar, la muestra de esta investigación estaba integrada fundamentalmente por mujeres, aunque,

4 Estas prácticas se basan en el modelo que presenta la American Psychological Association - http://www.apaexcellence.org reconocemos que estas constituyen la gran mayoría de quienes presentan esta enfermedad. La participación de una cantidad mayor de hombres hubiese permitido hacer una comparación de los resultados de ambos grupos.

Futuras investigaciones deben integrar muestras más grandes utilizando técnicas diversas de muestreo y recopilación de datos (cuantitativos y cualitativos) para conocer más a fondo el impacto de la fibromialgia en el contexto laboral. Resulta pertinente como futura línea de investigación la inclusión de otras variables, como los recursos personales, que han sido poco estudiadas en investigaciones cualitativas y cuantitativas sobre la fibromialgia (Bossema et al., 2012). En particular, es necesario prestar mayor atención a aspectos tales como las características personales y los recursos psicológicos positivos en pacientes con fibromialgia y otros dolores crónicos. En fin, es importante continuar estudiando la fibromialgia y sus efectos desde una mirada psicosocial con el propósito de aportar a la comprensión sobre la enfermedad, y sobre los mecanismos que podrían ayudar a aumentar la calidad de vida laboral de quienes la padecen.

\section{Referencias}

American College of Rheumatology (2017, marzo). Fibromialgia. Recuperado en https://www. rheumatology.org/I-Am-A/Patient-Caregiver/ Enfermedades-y Condiciones/Fibromialgia.

Avey, J., Reichard, R., Luthans, F., \& Mhatre, K. (2011). Meta-analysis of the impact of positive psychological capital on employee attitudes, behaviors and performance.

Human Resource Development Quarterly, 22, 127-152.

Bakker, A. B., \& Demerouti, E. (2013). La Teoría de las demandas y los recursos laborales. Journal of Work and Organizational Psychology, 29, 107-115.

Blasco, L., Mallo, M., Mencía, A., Franch, J., Casaus, P., Peña, J., ... \& Jariod, M. (2006). Perfiles clínicos en pacientes con fibromialgia que acuden a un centro de salud mental: obtención de un índice predictivo de gravedad psicopatológica. Actas Españolas de Psiquiatría, 34(2), 112-122.

Bossema, E. R., Kool, M.B., Cornet, D., Vermaas, P., 
de Jong M., van Middendorp, H., \& Geenen, R. (2012). Characteristics of suitable work from the perspective of patients with fibromyalgia. Rheumatology, 51, 311-318.

Briones, E., Ronda, E., \& Vives, C., (2014). Percepciones de pacientes con fibromialgia sobre el impacto de la enfermedad en el ámbito laboral. Atención Primaria, 47(4), 205-212.

Burckhardt, C. S., Clark, S. R., \& Bennett, R. M. (1993). Fibromyalgia and quality of life: A comparative analysis. Journal of Rheumatology, 20(3), 475-479.

Creswell, J. (2008). Research design: Qualitative, quantitative, and mixed methods approaches. (3rd ed.). USA: Sage Publication.

González, M., García, J., \& Landero, R. (2011). El papel de la teoría transaccional del estrés en el desarrollo de la fibromialgia: Un modelo de ecuaciones estructurales. Actas Españolas de Psiquiatría, 39(2), 81-87.

Guymer, E. K., Littlejohn, G. O., Brand, C. K., \& Kwiatek, R. A. (2016). Fibromyalgia onset has a high impact on work ability in Australians. Internal Medicine Journal, 46, 1069-1074.

Henriksson, C., Liedberg, G., \& Gerdle, B., (2005). Women with fibromyalgia: Work and rehabilitation. Disability and Rehabilitation, 27(12), 685-695.

Juuso, P., Skär, L., Sundin, K., \& Söderberg, S. (2016). The Workplace Experiences of Women with Fibromyalgia. Musculoskelet Care, 14, 69-76.

Kassam, A. \& Patten, S. (2006). Major depression, fibromialgia and labour force participation: A population-based cross-sectional study. $B M C$ Musculoskeletal Disorders, 7(4), 1-5.

Kelley, P., \& Clifford, P. (1997). Coping with Chronic Pain: Assessing Narrative Approaches. Social Worker, 42(3), 266-277.

Kool, M., \& Geenen, R. (2012). Loneliness in Patients with Rheumatic Diseases: The Significance of Invalidation and Lack of Social Support. The Journal of Psychology, 146(1-2), 229-241.

Lawrence, R., Felson, D., Helmick, C., Arnold, L., Choi, H., Deyo, R., ... \& National Arthritis Data Workgroup. (2008). Estimates of the prevalence of arthritis and other rheumatic conditions in the United States: Part II. Arthritis Rheumatology, 58, 26-35.

León, F., \& Loza, C. (2015). Prevalencia de fibromialgia en el distrito de Chiclayo. Revista Médica Herediana, 26(3), 147-159.

Liedberg, G., Burckhardt, C., Henriksson, C. (2006). Young women with fibromyalgia in the United States and Sweden: perceived difficulties during the first year after diagnosis. Disability and Rehabilitation, 28(19), 1177-1184.

Löfgren, M., Ekholm, J., \& Öhman, A. (2006). 'A constant struggle': Successful strategies of women in work despite fibromyalgia. Disability and Rehabilitation, 28(7), 447455.

Luthans, F. Y., Youssef, C. M., \& Avolio, B. J. (2007). Psychology capital: Developing the human competitive edge. New York, NY: Oxford University Press.

Luthans, F., Youssef-Morgan, C., Sweetman, D. S., \& Harms, P. D. (2012). Meeting the Leadership Challenge of Employee Well-Being Through Relationship PsyCap and Health PsyCap. Journal of Leadership \& Organizational Studies, 20(1), 118-133.

Mannerkorpi, K. \& Gard, G. (2012). Hinders for continued work among persons with fibromyalgia. BMC Musculoskeletal Disorders, 13(96), 1-8.

McDonald, M., da Costa, M., \& Ullman, S. (2011). Musculoskeletal pain in the workforce: the effects of back, arthritis, and fibromyalgia pain on quality of life and work productivity. Journal of Occupational and Environmental Medice, 53(7), 765-770.

Millán, C. (2005, Julio 3). Epidemia que no se puede controlar. El Nuevo Día, p. 6. National Institute of Arthritis and Musculoskeletal and Skin Diseases (2014, Julio 30). Questions and Answers about fibromialgia. Recuperado en http://www.niams.nih.gov/Health_Info/ Fibromyalgia/default.asp.

Oldfield, M., MacEachen, E., MacNeill, M., Kirsh, B. (2017). 'You want to show you're a valuable employee': A critical discourse analysis of multi-perspective portrayals of employed women with fibromyalgia. Chronic Illness, $0(0), 1-19$.

Palstam, A., Gard, G., \& Mannerkorpi, K. (2013). 
Factors promoting sustainable work in womenwith fibromyalgia. Disability and Rehabilitation, 35(19), 1622-1629.

Palstam, A., Larsson, A., Bjersing, J., Löfgren, M., Ernberg, M., Bileviciute ... \& Mannerkopi, K. (2014). Perceived Exertion at work in women with fibromyalgia: Explanatory factors and comparison with healthy women. Journal of Rehabilitation Medicine, 46, 773-780.

Palstam, A., \& Mannerkorpi, K. (2017). Work Ability in Fibromyalgia: An Update in 21 Century. Current Rheumatology Reviews, 13(2), 01-08.

Rakovski, C., Zettel, L., \& Rutledge, D. (2012). Association of employment and working conditions with physical and mental health symptoms for people with fibromyalgia. Disability \& Rehabilitation, 34(15), 12771283.

Rojas, A., Zapata, A., Anaya, J., \& Pineda, R. (2005). Predictores de calidad de vida en pacientes con fibromialgia. Revista Colombiana de Reumatología, 12(4), 295-300.

Salido, M., Navarro, P., Judez, E., \& Hortal, R. (2007). Factores relacionados con la incapacidad temporal en pacientes con fibromialgia. Reumatología Clínica, 3(2), 67-72.

Sallinen, M., Kukkurainen, M., Peltokallio, L., \& Mikkelsson, M. (2009). Women's narratives on experiences of work ability and functioning in fibromyalgia. Musculoskelet Care, 8, 1826.

Schaufeli, W. B., \& Taris, T.W. (2014). A critical review of the Job-Demands Resources Model: Implications for improving work and health. In G. Dauer \& O. Hammig. Bridging occupational, organizational and public health (p. 43-68). Dordrecht: Springer.

Van Wingerden, J., Derks, D., \& Bakker, A. (2015). The Impact of Personal Resources and Job Crafting Interventions on Work Engagement and Performance. Human Resource Management, 56(1), 51-67.

Valls, C. (2008). Diagnóstico diferencial del dolor y de la fibromialgia. Anuario de Psicología, 39(1), 87-92.

Vicente, M., Terradillos, M., Capdevila, L., Ramírez, M.V., \& López, A. (2011). Fibromialgia y Trabajo: Valoración en la legislación española.
Médica del Instituto Mexicano del Seguro Social, 49(5), 511-516.

Vilá, H. (2007). El dolor y su cronicidad en la teoría freudiana. El Amauta, 4, 1-14.

Xanthopoulou, D., Bakker, A., Demerouti, E., \& Schaufeli, W. (2007). The Role of Personal Resources in the Job Demands-Resources Model. International Journal of Stress Management, 14 (2), 121-141.

Youssef-Morgan, C. M., \& Luthans, F. (2015). Psychological Capital and Well-being. Stress Health, 31, 180-188. 